\title{
Shikonin induces apoptosis of HaCaT cells via the mitochondrial, Erk and Akt pathways
}

\author{
HUILING JING ${ }^{1,2^{*}}$, WENYAN SUN ${ }^{3,4^{*}}$, JINGHUA FAN ${ }^{1}$, YANMIN ZHANG ${ }^{5}$, JIAO YANG ${ }^{1}$, \\ JINJING JIA ${ }^{1}$, JICHANG LI ${ }^{1}$, JIAQI GUO ${ }^{1}$, SUJU LUO ${ }^{6}$ and YAN ZHENG ${ }^{1}$
}

\author{
${ }^{1}$ Department of Dermatology, The Second Affiliated Hospital of Xi'an Jiaotong University, Xi'an, Shaanxi 710004; \\ ${ }^{2}$ Department of Dermatology, Xi'an Hospital of Traditional Chinese Medicine, Xi'an, Shaanxi 710001; \\ ${ }^{3}$ Department of Physiology and Pathophysiology, Cardiovascular Research Center, School of Medicine, \\ Xi'an Jiaotong University; ${ }^{4}$ Department of Nutrition and Food Security, School of Public Health, \\ Xi'an Jiaotong University; ${ }^{5}$ School of Pharmacy, Health Science Center, Xi'an Jiaotong University, Xi'an, \\ Shaanxi 710061; ${ }^{6}$ Department of Dermatology, Tianjin Medical University General Hospital, Tianjin 300070, P.R. China
}

Received March 29, 2015; Accepted January 22, 2016

DOI: $10.3892 / \mathrm{mmr} .2016 .4917$

\begin{abstract}
Shikonin, which is a major ingredient of the traditional Chinese herb Lithospermum erythrorhizon, possesses various biological functions, including antimicrobial, anti-inflammatory, and antitumor activities. The present study aimed to determine the molecular mechanisms underlying the effects of shikonin on $\mathrm{HaCaT}$ cell apoptosis. Treatment with shikonin significantly inhibited the viability of $\mathrm{HaCaT}$ cells in a dose- and time-dependent manner, and promoted cell cycle arrest at $\mathrm{G}_{0} / \mathrm{G}_{1}$ phase and apoptosis. In addition, shikonin treatment reduced the mitochondrial membrane potential and induced reactive oxygen species generation. The results of a western blot analysis demonstrated that shikonin significantly activated caspase 3 expression, downregulated B-cell lymphoma 2 (Bcl-2) expression, and upregulated Bcl-2-associated $\mathrm{X}$ protein and $\mathrm{Bcl}-2$ homologous antagonist killer expression in a dose-dependent manner in $\mathrm{HaCaT}$ cells. Furthermore, shikonin decreased extracellular signal-regulated kinase (Erk) and Akt phosphorylation. These results indicated that shikonin may exert its anti-proliferative effects by inducing apoptosis via activation of the mitochondrial signaling pathway and inactivation of the Akt and Erk pathways in HaCaT cells. Therefore, the present study suggested that shikonin may have potential as a component of therapeutic strategies for the treatment of skin diseases.
\end{abstract}

Correspondence to: Professor Yan Zheng, Department of Dermatology, The Second Affiliated Hospital of Xi'an Jiaotong University, 157 Xiwu Road, Xi'an, Shaanxi 710004, P.R. China E-mail: zenyan66@126.com

*Contributed equally

Key words: shikonin, apoptosis, reactive oxygen species, Akt, extracellular signal-regulated kinase, $\mathrm{HaCaT}$ cells

\section{Introduction}

Psoriasis is a complex inflammatory skin disease that is characterized by inflammatory cell infiltration, increased dermal vascularity, and keratinocyte proliferation (1). The HaCaT cell line is an immortalized line of human epidermal keratinocytes, which has previously been used in experiments examining the effects of therapeutic drugs on keratinocytes (2). A series of clinical studies have indicated that certain Chinese herbs are effective in psoriasis treatment $(3,4)$. Shikonin, which is a naphthoquinone isolated from the Chinese herbal plant Lithospermum erythrorhizon, has long been used in traditional medicine to treat hemorrhoids, burns, infected wounds, anal ulcers, external wounds, and psoriasis $(5,6)$.

Shikonin is known to possess several medicinal properties, including promotion of wound healing, and antibacterial, anti-inflammatory and antitumor effects (5). In addition, shikonin has been shown to exert inhibitory effects on tumor necrosis factor- $\alpha$-induced angiogenesis, tumor cell-induced angiogenesis, and normal programmed developmental angiogenesis (7). Shikonin also inhibits angiogenesis in inflammatory skin diseases, such as psoriasis (8). Shikonin treatment has been reported to activate the caspase pathway, in order to induce cellular apoptosis in HL-60 leukemia cells (9) and human colorectal cancer cells (10). Shikonin induces apoptosis via the reactive oxygen species (ROS)/extracellular signal-regulated kinase (Erk) pathway in osteosarcoma cells (11). Furthermore, it inhibits cell proliferation by decreasing Erk activities in human epidermoid carcinoma cells (12), and triggers apoptosis through the ROS/Akt and nuclear factor- $\kappa \mathrm{B}(\mathrm{NF}-\kappa \mathrm{B})$ pathways in hepatocellular carcinoma cells (13). Shikonin also elevates ROS generation to induce apoptosis in human glioma cells (14), and is able to increase intracellular ROS generation during the early phase of apoptotic progression, alongside a disturbance in mitochondrial transmembrane potential, in SK-Hep-1 hepatoma cells (15).

Two apoptotic pathways have been established: The cell death receptor pathway and the mitochondria-initiated 
pathway (16). Members of the B-cell lymphoma 2 (Bcl-2) family are key regulators of mitochondria-initiated apoptosis. When anti-apoptotic members of the Bcl-2 family are inhibited and/or proapoptotic members are activated, mitochondrial integrity is disrupted and cytochrome $c$ is released into the cytosol (17), thus activating caspase 9 and caspase 3 , and subsequently leading to cell apoptosis (18). The present study aimed to investigate whether shikonin was able to induce mitochondrial-initiated apoptosis in $\mathrm{HaCaT}$ cells, in order to inhibit cell proliferation.

\section{Materials and methods}

Chemicals and reagents. Shikonin was purchased from Shanghai PureOne Biotechnology Co., Ltd. (Shanghai, China), and its purity was determined to be $\sim 99.5 \%$ using high-performance liquid chromatography. Cell culture medium (RPMI-1640), trypsin, 3-(4,5-dimethyl-2-thiazolyl) -2,5-diphenyl-2H-tetrazolium bromide (MTT), Hoechst 33258 and dimethyl sulfoxide (DMSO) were purchased from Sigma-Aldrich (St. Louis, MO, USA). RNase, propidium iodide (PI), Annexin V-fluorescein isothiocyanate (FITC), ROS and 5,5',6,6'-tetrachloro-1,1',3,3'-tetraethylbenzimidazolylcarbocyanine iodide (JC-1) were purchased from Nanjing KeyGen Biotech Co., Ltd. (Nanjing, China). Fetal bovine serum (FBS) was purchased from National Hyclone (Lanzhou) Bio-engineering Co., Ltd. (Lanzhou, China). Rabbit polyclonal antibodies against caspase 3 (9662), Akt (9272), phosphorylated (p)-Akt (9271), Erk1/2 (9102) and p-Erk1/2 (9101) were purchased from Cell Signaling Technology, Inc. (Danvers, MA, USA). Antibodies against cyclin B1 (55004-1-AP), cyclin D1 (60186-1-Ig), cyclin E (11554-1-AP), Bcl-2 (12789-1-AP), Bcl-2-associated X protein (Bax; 50599-2-Ig) and Bcl-2 homologous antagonist killer (Bak; 14673-1-AP) were purchased from Proteintech Group, Inc. (Rosemont, IL, USA), and antibodies against glyceraldehyde 3-phosphate dehydrogenase (GAPDH; sc-365062) and $\beta$-actin (sc-47778) were purchased from Santa Cruz Biotechnology, Inc. (Dallas, TX, USA). Horseradish peroxidase (HRP)-conjugated goat anti-rabbit IgG $(\mathrm{H}+\mathrm{L})$ secondary antibodies (A0208) were purchased from Beyotime Institute of Biotechnology, Nanjing, China).

Cell culture. The HaCaT normal human epidermal keratinocyte cell line was obtained from the Chinese Academy of Sciences (Kunming, China). The cells were cultured in RPMI-1640 supplemented with 10\% FBS and 1\% penicillin -streptomycin (Hyclone; GE Healthcare Life Sciences, Logan, UT, USA) at $37^{\circ} \mathrm{C}$ in an atmosphere containing $5 \%$ $\mathrm{CO}_{2}$.

Cell viability assay. Cell viability was determined using the MTT colorimetric assay. Exponentially growing cells were seeded in 96-well plates in culture medium at density of $2 \times 10^{4}$ cells/well. Following a $24 \mathrm{~h}$ incubation, the cells were treated with various concentrations of shikonin between 0-20 $\mu \mathrm{M}$ for 24 and $48 \mathrm{~h}$. Subsequently, the medium was discarded, and $200 \mu \mathrm{l}$ MTT $(0.5 \mathrm{mg} / \mathrm{ml})$ was added to each well and incubated for $4 \mathrm{~h}$ at $37^{\circ} \mathrm{C}$. The medium was then removed, and the formazan salt was dissolved in $150 \mu \mathrm{l}$
DMSO. Optical density of the cells was determined using a Bio-Rad Model 680 microplate reader (Bio-Rad Laboratories, Inc., Hercules, CA, USA) at a wavelength of $490 \mathrm{~nm}$. Cell viability was expressed as a percentage of the control. Three replicate wells were used for each analysis.

Cell cycle analysis using flow cytometry. Exponentially growing cells were seeded in 6-well culture plates in culture medium at a density of $4 \times 10^{5}$ cells/well. Following treatment with 1,2 and $4 \mu \mathrm{M}$ shikonin for $24 \mathrm{~h}$, both adherent and floating cells were collected, washed in ice-cold phosphate-buffered saline (PBS), and fixed with ice-cold $70 \%$ ethanol overnight. After fixation, the ethanol was removed via centrifugation, and the cells were suspended in $0.1 \mathrm{ml}$ RNase solution at $37^{\circ} \mathrm{C}$ for $30 \mathrm{~min}$. Subsequently, $0.4 \mathrm{ml}$ PI was added and incubated at $4^{\circ} \mathrm{C}$ for $30 \mathrm{~min}$ in the dark. Stained cells were analyzed using a FACSCanto flow cytometer (BD Biosciences, San Jose, CA, USA). Data acquisition and analyses were performed using WinMDI 2.9 software (BD Biosciences).

DNA morphological observation using Hoechst staining assay. To visualize apoptotic cell death and nuclear morphology, the cells were stained with Hoechst 33258. Briefly, the treated cells were collected, washed twice in PBS, and fixed in $4 \%$ formaldehyde for $10 \mathrm{~min}$. The cells were then washed and stained with Hoechst 33258 for 20 min at room temperature, after which they were examined under a Nikon Eclipse TE2000-PFS inverted fluorescence microscope (Nikon Corporation, Tokyo, Japan) at $340 \mathrm{~nm}$. The number of apoptotic cells was measured by calculating the percentage of cells displaying chromatin condensation compared with the total number of cells.

Detection of apoptosis using flow cytometry. Cellular apoptosis was detected using an Annexin V-FITC/PI Apoptosis Detection kit. Briefly, HaCaT cells were treated with 1,2 and $4 \mu \mathrm{M}$ shikonin for $24 \mathrm{~h}$, and collected via centrifugation. The cells were then washed in PBS and resuspended in binding buffer, and the apoptotic cell death rate was examined using Annexin V-FITC and PI double staining (incubation with $5 \mu \mathrm{l}$ Annexin V-FITC and $10 \mu \mathrm{l}$ PI for $15 \mathrm{~min}$ in the dark), according to the manufacturer's protocol. Subsequently, the cells stained with Annexin V-FITC/PI were detected using a FACSCanto flow cytometer (BD Biosciences). Data acquisition and analyses were performed using WinMDI 2.9 software. All experiments were performed in triplicate.

Measurement of the mitochondrial membrane potential $(\Delta \psi m)$. The $\Delta \psi \mathrm{m}$ was assessed as previously described (19). Briefly, the HaCaT cells were treated with 1,2 and $4 \mu \mathrm{M}$ shikonin for $4 \mathrm{~h}$, and were harvested and collected via centrifugation. The cells were resuspended in PBS and were then incubated with $10 \mu \mathrm{M} \mathrm{JC}-1$ for $15 \mathrm{~min}$ at room temperature in the dark. The fluorescently labeled cells were washed in PBS and analyzed using a BD FACSCalibur flow cytometry system (excitation, $485 \mathrm{~nm}$; emission, 530/590 nm; BD Biosciences). The $590 \mathrm{~nm} / 530 \mathrm{~nm}$ fluorescence ratio was used to quantify the $\Delta \psi \mathrm{m}$. Data acquisition and analyses were performed using WinMDI 2.9 software. 
Intracellular ROS assay. The ROS generation assay was performed as described in our previous study (20). Briefly, the $\mathrm{HaCaT}$ cells were treated with 1,2 and $4 \mu \mathrm{M}$ shikonin for $24 \mathrm{~h}$, and were harvested and collected via centrifugation. The cells were resuspended in PBS and were then incubated with $10 \mu \mathrm{M} \mathrm{H}_{2}$ DCF-DA (Nanjing KeyGen Biotech Co., Ltd.) for $30 \mathrm{~min}$ at room temperature in the dark. The fluorescently labeled cells were washed in PBS and analyzed using a BD FACSCalibur flow cytometry system (excitation, $485 \mathrm{~nm}$; emission, $538 \mathrm{~nm}$; BD Biosciences). Data acquisition and analyses were performed using WinMDI 2.9 software.

Western blot analysis. HaCaT cells, treated with 1, 2 and $4 \mu \mathrm{M}$ shikonin for $24 \mathrm{~h}$, were lysed in radioimmunoprecipitation assay lysis buffer containing a protease and phosphatase inhibitor cocktail on ice for $30 \mathrm{~min}$. Then lysate was then collected and centrifuged at 56,000 $\mathrm{x}$ g for $15 \mathrm{~min}$ at $4^{\circ} \mathrm{C}$. Protein concentration was determined using the Bicinchoninic Acid Protein Quantification kit (Pierce Biotechnology, Inc., Rockford, IL, USA), according to the manufacturer's protocol. Protein lysates were then denatured for $10 \mathrm{~min}$ at $95^{\circ} \mathrm{C}$ and $50 \mu \mathrm{g}$ protein per lane was separated by $10 \%$ sodium dodecyl sulfate-polyacrylamide gel electrophoresis and transferred to polyvinylidene difluoride (PVDF) membranes (EMD Millipore, Billerica, MA, USA). After immunoblotting, the PVDF membranes were blocked with $5 \%$ skimmed milk for at least $2 \mathrm{~h}$, washed and incubated with anti-caspase 3, anti-Akt, anti-p-Akt, anti-Erk 1/2, anti-p-Erk 1/2, anti-cyclin B1, anti-cyclin D1, anti-cyclin E, anti-Bcl-2, anti-Bax, anti-Bak, anti-GAPDH and anti- $\beta$-actin primary antibodies at $4^{\circ} \mathrm{C}$ overnight (all 1:1,000). Subsequently, the membranes were washed three times with Tris-buffered saline with Tween 20 and incubated with the HRP-conjugated goat anti-rabbit $\operatorname{IgG}(\mathrm{H}+\mathrm{L})$ secondary antibodies $(1: 10,000)$ at $37^{\circ} \mathrm{C}$ for $1 \mathrm{~h}$. Chemiluminescence detection was assayed using an enhanced chemiluminescence detection kit (Pierce Biotechnology, Inc.). Results were analyzed using Quantity One software (version 4.4.0.36; Bio-Rad Laboratories, Inc.), in order to obtain the optical density ratio of the target protein to GAPDH and $\beta$-actin.

Statistical analysis. All of the data, which were obtained from at least three independent experiments, were expressed as the mean \pm standard deviation for each group. Statistical analyses, including Student's t-test, one-way analysis of variance and regression analysis, were performed using GraphPad Prism 4.0 software (GraphPad, Inc., La Jolla, CA, USA). P<0.05 was considered to indicate a statistically significant difference.

\section{Results}

Shikonin inhibits the growth of HaCaT cells. The chemical structure of shikonin is shown in Fig. 1A (21). The effects of shikonin on the growth of $\mathrm{HaCaT}$ cells were evaluated using the MTT assay. Human HaCaT cells were treated with various concentrations of shikonin $(0-20 \mu \mathrm{M})$ for 24 and $48 \mathrm{~h}$. As shown in Fig. 1B, the proliferation of shikonin-treated $\mathrm{HaCaT}$ cells was markedly suppressed at 24 and 48 h compared with the control group. These results suggest that shikonin may exhibit dose- and time-dependent inhibitory effects on the viability of $\mathrm{HaCaT}$ cells. The calculated half maximal inhibitory concentration
A

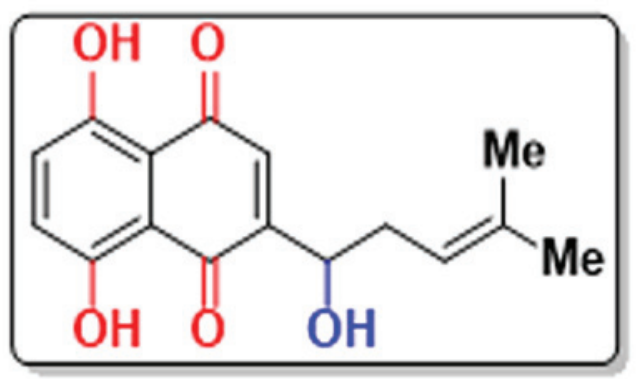

B

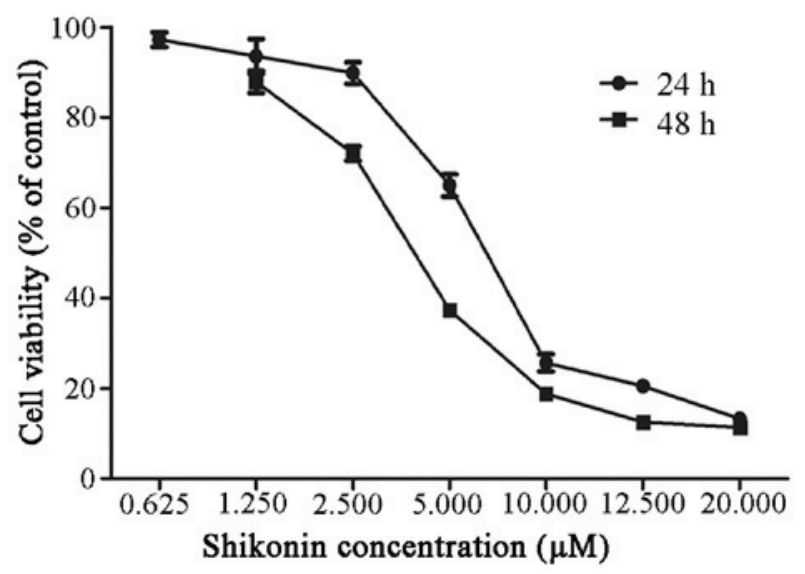

Figure 1. Effects of shikonin on human HaCaT cell viability. HaCaT cells were treated with various concentrations of shikonin for 24 and $48 \mathrm{~h}$. (A) Chemical structure of shikonin. (B) MTT assay was performed to select the appropriate doses of shikonin for subsequent experiments. Data are presented as the mean \pm standard deviation obtained from three independent experiments.

$\left(\mathrm{IC}_{50}\right)$ values for shikonin were 6.34 and $2.43 \mu \mathrm{M}$ at 24 and $48 \mathrm{~h}$, respectively. Based on these $\mathrm{IC}_{50}$ values, doses of 1,2 and $4 \mu \mathrm{M}$ shikonin were selected for use in subsequent experiments to assess HaCaT cell growth inhibition. Notably, concentrations of DMSO, which was used to dissolve shikonin, were maintained at $<0.2 \%(\mathrm{v} / \mathrm{v})$.

Shikonin induces a cell cycle arrest at $G_{0} / G_{1}$ phase in HaCaT cells. To investigate the mechanisms underlying shikonin-induced inhibition of cell proliferation, changes in cell cycle progression were detected after shikonin treatment using flow cytometry. The percentage of cells that accumulated in $G_{0} / G_{1}$ phase was significantly increased following treatment with 2 and $4 \mu \mathrm{M}$ shikonin for $24 \mathrm{~h}$, as compared with the control group $(\mathrm{P}<0.05$ and $\mathrm{P}<0.01$, respectively; Fig. $2 \mathrm{~A}$ and $\mathrm{B})$. These results indicate that shikonin may partially mediate $\mathrm{HaCaT}$ cell growth inhibition by inducing $\mathrm{G}_{0} / \mathrm{G}_{1}$ phase cell cycle arrest.

Following a $24 \mathrm{~h}$ treatment with 1,2 or $4 \mu \mathrm{M}$ shikonin, the expression levels of cell cycle regulatory proteins were examined in HaCaT cells using western blot analysis. As shown in Fig. 2C, cyclin D1 expression was significantly increased following treatment with 2 and $4 \mu \mathrm{M}$ shikonin, as compared with the control group $(\mathrm{P}<0.01$ and $\mathrm{P}<0.001$, respectively); whereas cyclin $\mathrm{B} 1$ and cyclin $\mathrm{E}$ expression levels were decreased in a dose-dependent manner (Fig. 2C and D). 
A
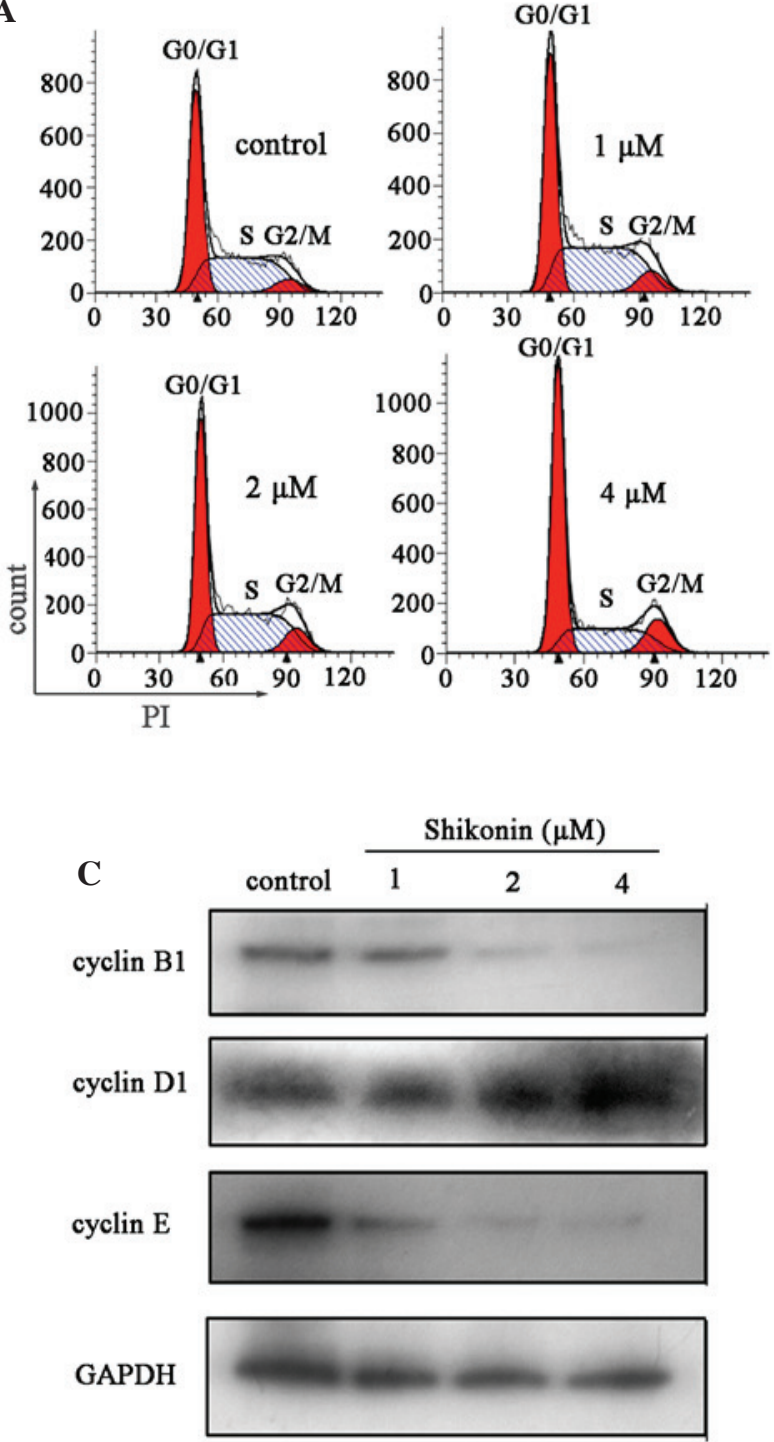

B

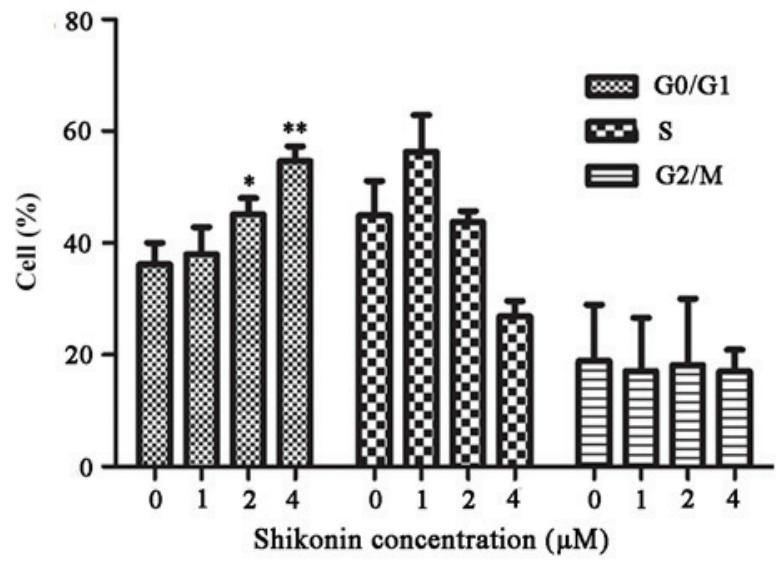

D

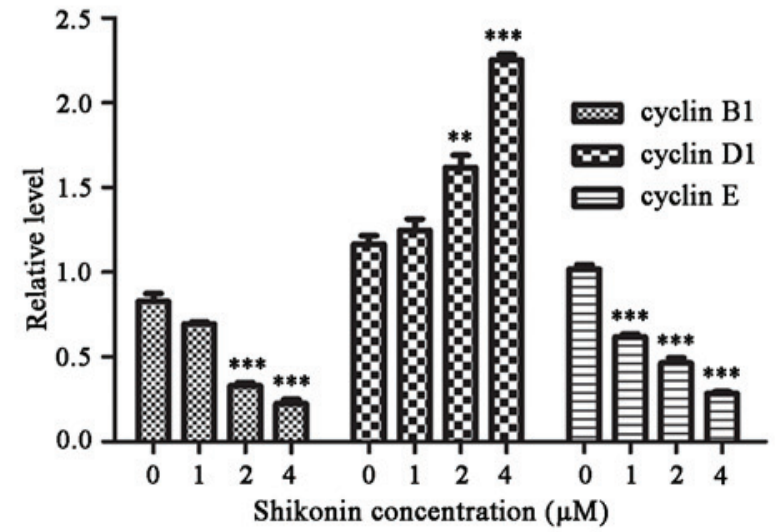

Figure 2. Effects of shikonin on cell cycle progression and the expression levels of cell cycle regulatory proteins. HaCaT cells were treated with 1,2 or $4 \mu \mathrm{M}$ shikonin for $24 \mathrm{~h}$. (A and B) Flow cytometric analysis was used to determine cell cycle progression, and cell cycle profiles were analyzed to quantify cell cycle distribution. (C and D) Western blot analysis of cyclin B1, cyclin D1 and cyclin E protein expression. Data are presented as the mean \pm standard deviation obtained from three independent experiments. ${ }^{*} \mathrm{P}<0.05,{ }^{* *} \mathrm{P}<0.01$ and ${ }^{* * *} \mathrm{P}<0.001$ vs. the control group. PI, propidium iodide; GAPDH, glyceraldehyde 3-phosphate dehydrogenase.

Shikonin induces apoptosis of HaCaT cells. To assess whether shikonin-induced cell growth inhibition was associated with cell apoptosis, the effects of shikonin on apoptosis were evaluated by flow cytometry using Annexin V-FITC/PI double staining. As shown in Fig. 3A, the percentage of dead cells (Annexin V-positive, PI-positive) was increased in a dose-dependent manner. The percentage of apoptotic HaCaT cells following a $24 \mathrm{~h}$ treatment with 2 or $4 \mu \mathrm{M}$ shikonin was 22.0 and $33.6 \%$, respectively; therefore, the percentage of apoptotic cells were significantly increased, as compared with the control group $(\mathrm{P}<0.05$ and $\mathrm{P}<0.01$, respectively; Fig. $3 \mathrm{~A}$ and $\mathrm{B})$. These results correspond to the addition of values that are shown in the higher and lower right quadrants of each panel, which indicate the early and late stages of apoptosis, respectively.

Nuclear fragmentation is an important characteristic of apoptosis, which is easily distinguished by Hoechst staining. As shown in Fig. 3C, marked nuclear condensation or nuclear fragmentation was induced following treatment of the cells with
2 or $4 \mu \mathrm{M}$ shikonin for $24 \mathrm{~h}$. These results clearly indicate that inducing cellular apoptosis is a primary mechanism underlying the inhibitory effects of shikonin on $\mathrm{HaCaT}$ cell growth. In addition, shikonin-induced apoptosis occurred in a dose-dependent manner.

Shikonin decreases the $\triangle \psi m$ and induces ROS generation. To evaluate whether the mitochondrial pathway was responsible for shikonin-induced apoptosis, the effects of shikonin on the $\Delta \psi \mathrm{m}$ after $4 \mathrm{~h}$ were examined using the mitochondria-specific dye, JC-1. The percentage of cells with depolarized $\Delta \psi \mathrm{m}$ significantly increased following treatment of $\mathrm{HaCaT}$ cells with 1,2 or $4 \mu \mathrm{M}$ shikonin for $4 \mathrm{~h}$, as compared with the untreated cells, and this trend occurred in a dose-dependent manner $(\mathrm{P}<0.05$ and $\mathrm{P}<0.01$, respectively; Fig. 4A and B). Depolarization of the $\Delta \psi \mathrm{m}$ is a characteristic event of early apoptosis.

Since intracellular ROS generation is considered to be associated with mitochondrial dysfunction, the present study further 
A
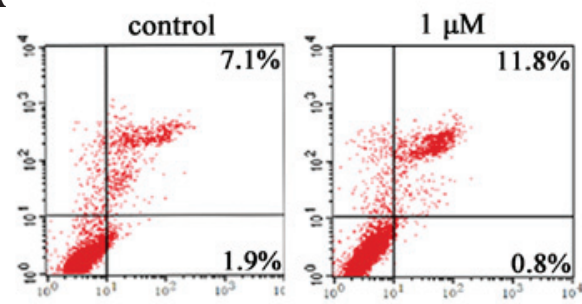

$2 \mu \mathrm{M}$

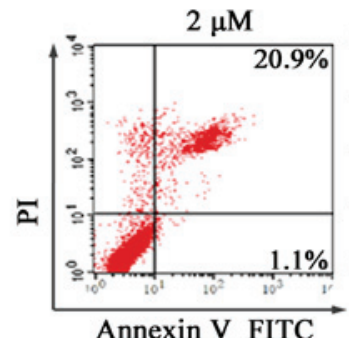

Annexin V FITC
$4 \mu \mathrm{M}$

$.4 \%$

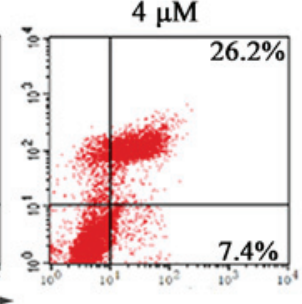

C

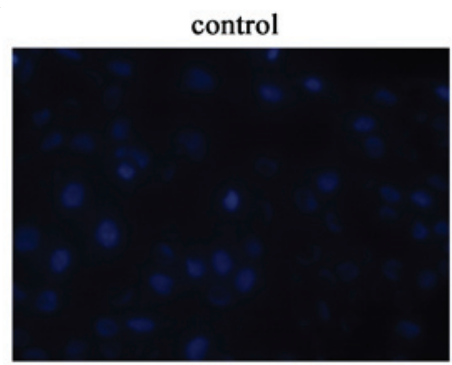

$2 \mu \mathrm{M}$

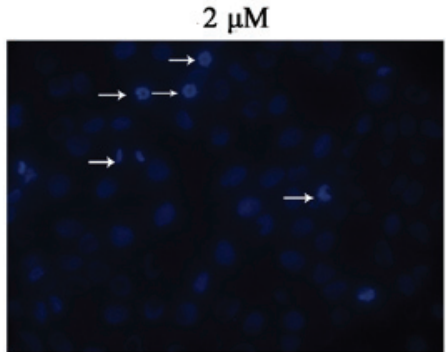

B

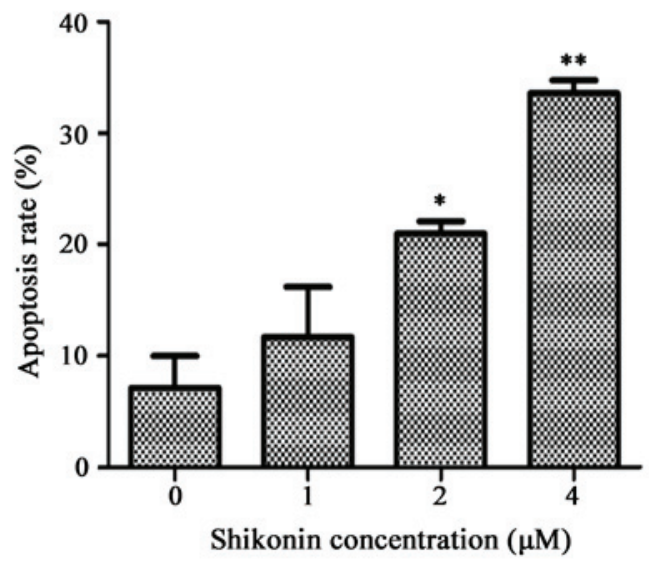

$1 \mu \mathrm{M}$

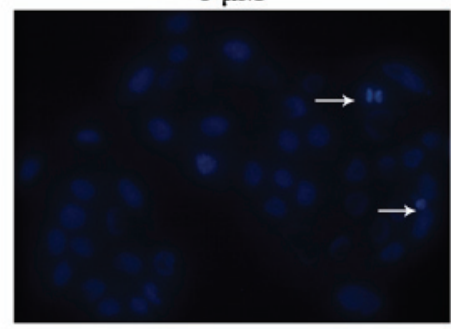

$4 \mu \mathrm{M}$

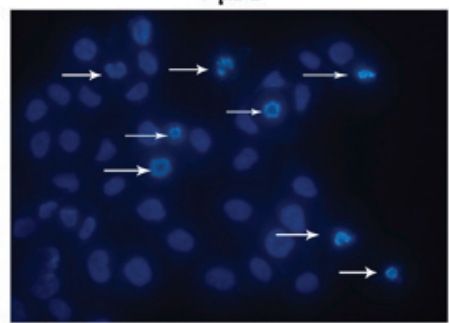

Figure 3. Effects of shikonin on cell apoptosis. HaCaT cells were treated with 1, 2 or $4 \mu \mathrm{M}$ shikonin for $24 \mathrm{~h}$. (A and B) Analysis of HaCaT cell apoptosis. The treated cells were stained with Annexin V/propidium iodide (PI) and were subjected to flow cytometry. (C) Apoptosis-related morphological changes were observed via fluorescence microscopy using Hoechst staining (magnification, 40x). The apoptotic cells displayed condensed and fragmented nuclei and reduced cell volumes in a dose-dependent manner. Data are presented as the mean \pm standard deviation obtained from three independent experiments. Arrows indicate cells with nuclear condensation or fragmentation ${ }^{*} \mathrm{P}<0.05$ and ${ }^{* *} \mathrm{P}<0.01$ vs. the control group. FITC, fluorescein isothiocyanate.

examined whether shikonin could stimulate ROS generation in $\mathrm{HaCaT}$ cells. A significant increase in ROS generation was observed in the HaCaT cells treated with shikonin compared with the untreated cells ( $\mathrm{P}<0.001$; Fig. 4C and D).

Shikonin regulates Bcl-2 family members and activates caspase family proteins. Bcl-2 family proteins are known to be involved in the apoptotic process, due to their ability to form membrane channels in mitochondria. The Bcl-2 proteins control cytochrome $c$ release into the cytosol, which activates caspase 3, subsequently leading to cell apoptosis (22). Therefore, the present study examined the expression levels of $\mathrm{Bcl}-2$ family proteins (Bcl-2, Bax and Bak), and caspase 3 in $\mathrm{HaCaT}$ cells treated with 1,2 or $4 \mu \mathrm{M}$ shikonin for $24 \mathrm{~h}$ using western blotting. The effects of shikonin on the expression levels of Bcl-2 family proteins are presented in Fig. 5A and B. Shikonin significantly decreased the expression levels of the anti-apoptotic Bcl-2 protein, and increased the expression levels of the proapoptotic Bax and Bak proteins in $\mathrm{HaCaT}$ cells, as compared with the untreated control cells. A dose-dependent significant increase in the Bax/Bcl-2 ratio was observed $(\mathrm{P}<0.001)$. The present study also verified the induction of apoptosis via caspase 3 activation using western blotting. The results revealed that treatment with shikonin significantly increased caspase 3 cleavage, as compared with the untreated cells $(\mathrm{P}<0.001$; Fig. $5 \mathrm{C}$ and $\mathrm{D})$. These results indicate that shikonin-induced $\mathrm{HaCaT}$ cell apoptosis is mediated via a mitochondria-dependent pathway.

Erk and Akt pathways are associated with shikonin-induced HaCaT cell apoptosis. The mitogen-activated protein kinases (MAPKs) and phosphatidylinositol 3-kinase (PI3K)/Akt pathways have essential roles in regulating cell proliferation, cell 
A

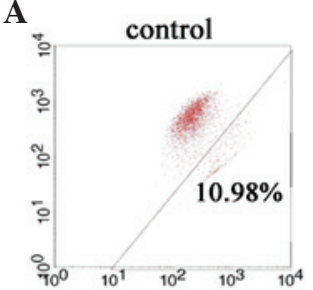

$2 \mu \mathrm{M}$

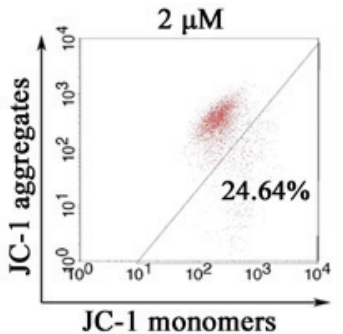

C

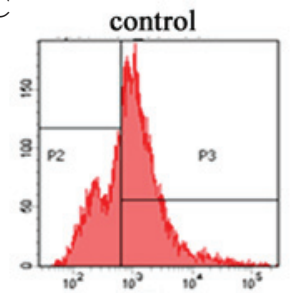

$2 \mu \mathrm{M}$

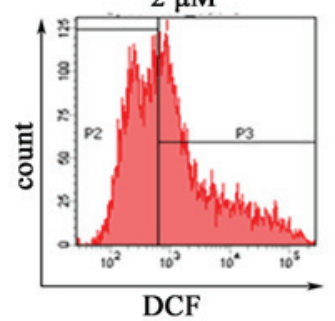

$1 \mu \mathrm{M}$

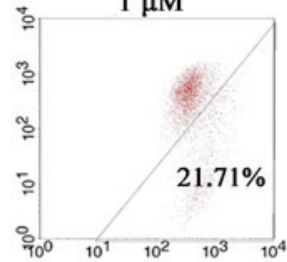

$4 \mu \mathrm{M}$

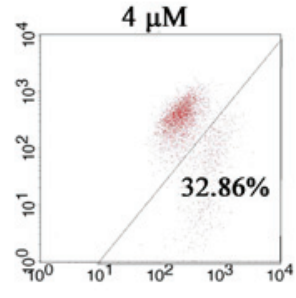

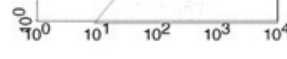

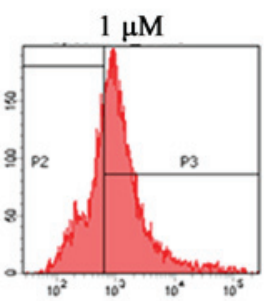

$4 \mu \mathrm{M}$

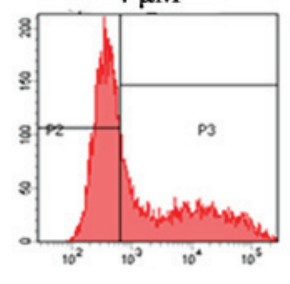

B

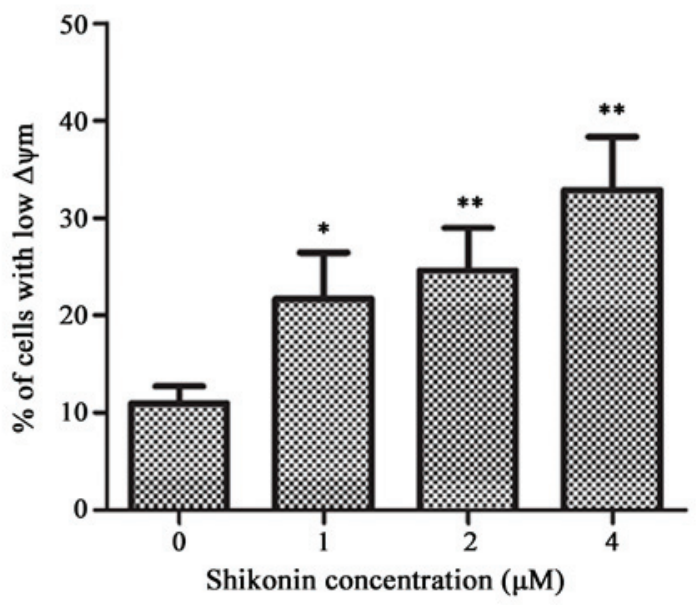

D

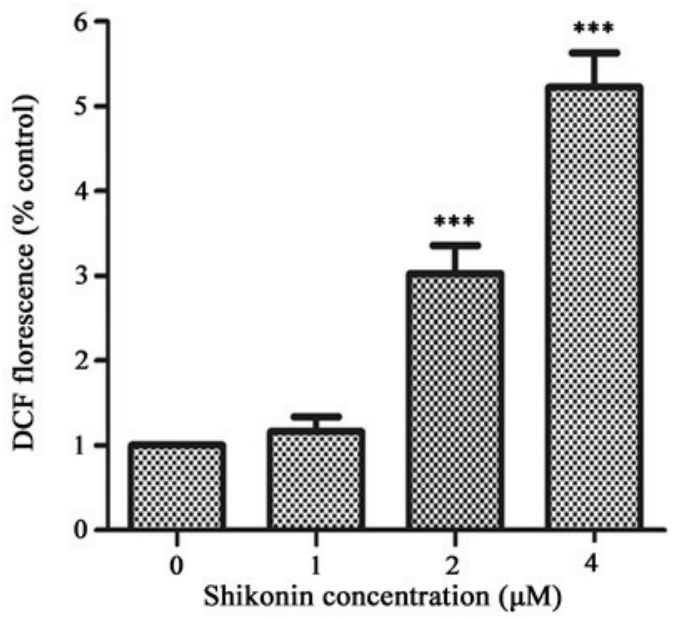

Figure 4. Effects of shikonin on the mitochondrial membrane potential $(\Delta \psi \mathrm{m})$ and induction of reactive oxygen species (ROS) generation. (A and B) $\Delta \psi \mathrm{m}$ was assessed using flow cytometry following treatment of the HaCaT cells with 1, 2 or $4 \mu \mathrm{M}$ shikonin for $24 \mathrm{~h}$. (C and D) ROS generation was assessed by flow cytometry following treatment of the HaCaT cells with 1,2 and $4 \mu \mathrm{M}$ shikonin for $24 \mathrm{~h}$. Data are presented as the mean \pm standard deviation obtained from three independent experiments. ${ }^{*} \mathrm{P}<0.05,{ }^{* *} \mathrm{P}<0.01$ and ${ }^{* * *} \mathrm{P}<0.001$ vs. the control group. DCF, dichlorofluorescein.

survival and apoptosis. To evaluate whether shikonin is capable of modulating the PI3K/Akt and MAPK signaling pathways in order to induce the inhibition of $\mathrm{HaCaT}$ cell proliferation, the effects of shikonin on the phosphorylation of signaling molecules in these two pathways were detected. As shown in Fig. 5E and $\mathrm{F}$, treatment of $\mathrm{HaCaT}$ cells with 1,2 or $4 \mu \mathrm{M}$ shikonin for $24 \mathrm{~h}$ led to a significant reduction in the expression levels of p-Erk1/2 and p-Akt, as compared with the total protein expression levels of Erk1/2 and Akt $(\mathrm{P}<0.001)$. These results suggest that shikonin may downregulate the phosphorylation of these proteins in a dose-dependent manner.

\section{Discussion}

HaCaT cells have been used extensively as in vitro models of psoriasis $(2,23,24)$. Apoptotic inhibition occurs in psoriatic lesional keratinocytes (25), resulting in keratinocyte hyperproliferation, which induces psoriasis. Therefore, the present study hypothesized that effective therapeutic agents for the treatment of psoriasis should inhibit keratinocyte hyperproliferation and induce apoptosis.

The results of the present study revealed that shikonin significantly decreased $\mathrm{HaCaT}$ cell viability and induced a $\mathrm{G}_{0} / \mathrm{G}_{1}$ phase cell cycle arrest. These results indicated that cell cycle arrest may be partially responsible for shikonin-induced $\mathrm{HaCaT}$ cell growth inhibition. Phosphatidylserine is translocated from the inner to the outer leaflet of the plasma membrane in apoptotic cells. In the present study, Annexin V-FITC/PI staining was used to determine whether apoptosis had occurred. Compared with untreated cells, the fluorescence intensity of $\mathrm{HaCaT}$ cells treated with shikonin was significantly increased in a dose-dependent manner, which was indicative of apoptosis. These findings are similar to those from a previous report, which demonstrated that apoptosis of $\mathrm{HaCaT}$ human keratinocytes can be induced by celastrol, which is a triterpenoid isolated from Celastrus orbiculatus, via inhibition of NF- $\mathrm{BB}$ activity (24). 
$\mathbf{A}$

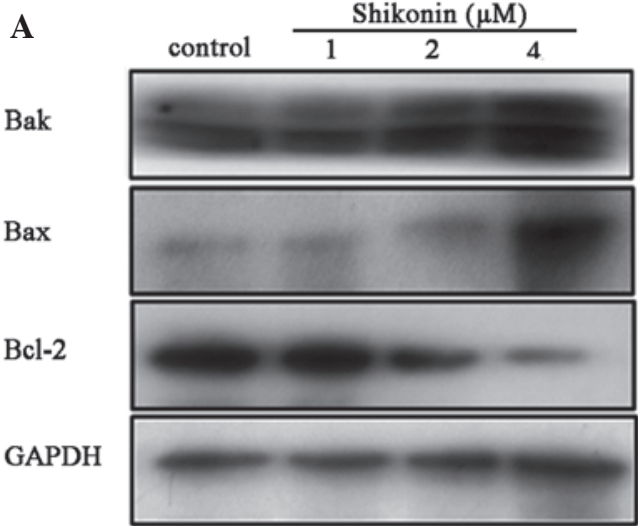

C

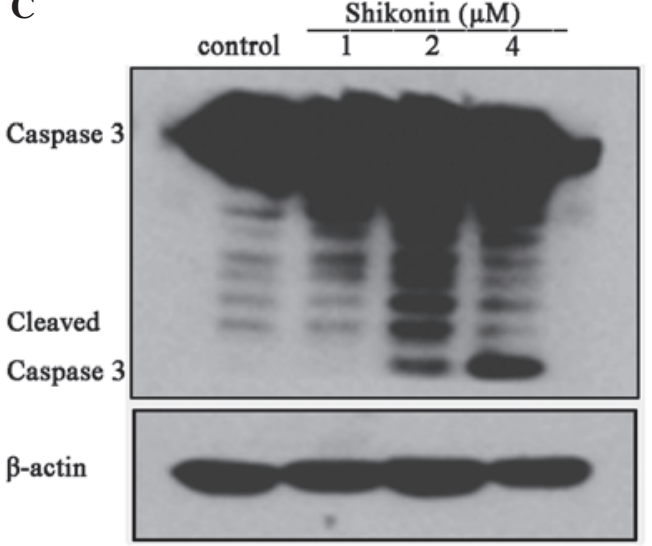

$\mathbf{E}$

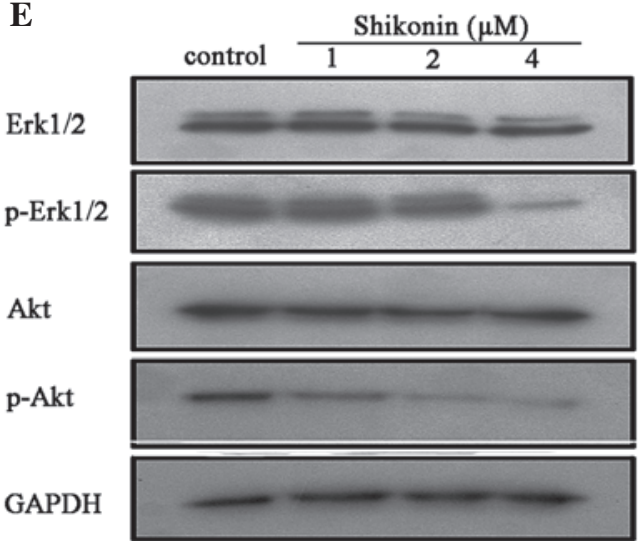

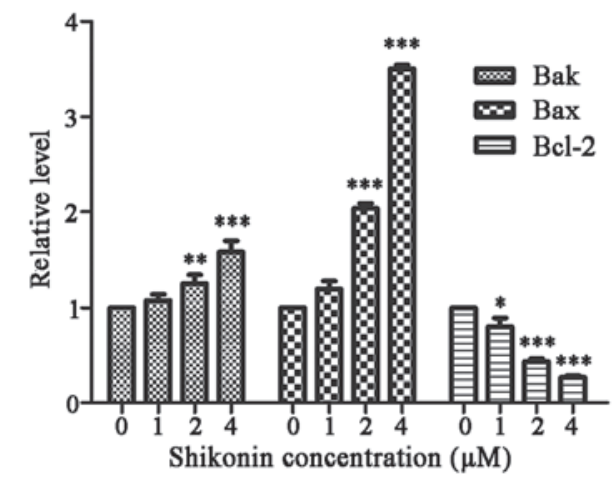

D

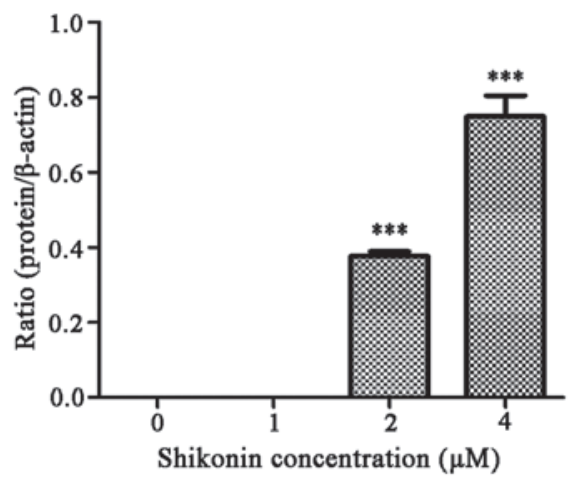

$\mathbf{F}$

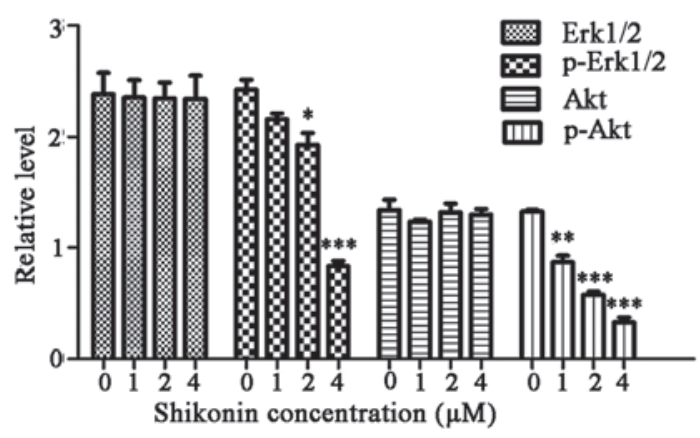

Figure 5. Effects of shikonin on the molecular expression of apoptotic-associated proteins and signaling pathways. HaCaT cells were treated with 1,2 or $4 \mu \mathrm{M}$ shikonin for $24 \mathrm{~h}$. (A-D) B-cell lymphoma 2 (Bcl-2) family proteins: Bcl-2, Bcl-2-associated X protein (Bax) and Bcl-2 homologous antagonist killer (Bak), and caspase 3 were assessed by western blotting. (E and F) Extracellular signal-regulated kinase (Erk) and Akt molecular pathways were assessed by western blotting. Data are presented as the mean \pm standard deviation obtained from three independent experiments. ${ }^{*} \mathrm{P}<0.05,{ }^{* *} \mathrm{P}<0.01$ and ${ }^{* * *} \mathrm{P}<0.001 \mathrm{vs}$. the control group. p-, phosphorylated; GAPDH, glyceraldehyde 3-phosphate dehydrogenase.

Apoptosis is a highly regulated process leading to programmed cell death, which is regulated by several signaling pathways, including the caspase and MAPK pathways (26). The $\mathrm{Bcl}-2$ protein family has an important role in the mitochondrial apoptotic pathway, which results in the release of mitochondrial cytochrome $c$, leading to caspase 9 activation and subsequent caspase 3 activation (27). The present study examined the effects of shikonin on mitochondrial function. The results demonstrated that the $\Delta \psi \mathrm{m}$ was significantly decreased, which was accompanied by an increase in ROS generation, indicating that mitochondrial dysfunction had occurred. Subsequently, an increase in caspase 3, Bax and Bak protein expression, and a decrease in Bcl-2 protein expression was observed in the shikonin-treated $\mathrm{HaCaT}$ cells, thus suggesting that shikonin-induced apoptosis occurred via the mitochondrial apoptotic pathway. 
Previous studies have demonstrated that shikonin potently inhibits cell growth and induces cell apoptosis in various types of cells via its effects on several molecular targets, including members of the MAPK family (28), Akt/apoptosis signal-regulating kinase 1/p38 (29) and NF- $\mathrm{B}$ (30). The present study evaluated the effects of shikonin on the phosphorylation of signaling molecules in these pathways in HaCaT cells. Treatment with shikonin resulted in marked reductions in the expression levels of p-Erk1/2 and p-Akt in a dose-dependent manner, thus suggesting that shikonin may dose-dependently downregulate the phosphorylation of these proteins.

In conclusion, in $\mathrm{HaCaT}$ human epidermal keratinocyte cells, shikonin is able to exert its anti-proliferative activity by inducing cellular apoptosis via the mitochondrial apoptotic pathway. The mechanism underlying these effects is associated with inactivation of the Akt and Erk pathways. Therefore, the present study suggested that shikonin may have potential as a component of therapeutic strategies for the treatment of skin diseases.

\section{Acknowledgements}

The present study was supported by the National Natural Science Foundation of China (grant nos. 81371732 and 81201231), and was partially supported by the Fundamental Research Funds for the Central Universities, the Key Fund and Medical Technology of Second Hospital of Xi'an Jiaotong University, and the Program for Changjiang Scholars and Innovative Research Team in University (grant no. PCSIRT:1171). The study was also supported by the Fundamental Research Funds of Xi'an Bureau of Public Health (grant no. J2014025) and the Xi'an Hospital of Traditional Chinese Medicine (grant no. 2014G01). The present study was performed in the laboratory of Professor Langchong He (School of Pharmacy, Xi'an Jiaotong University), and the authors thank him for his help and support.

\section{References}

1. Bhalerao J and Bowcock AM: The genetics of psoriasis: A complex disorder of the skin and immune system. Hum Mol Genet 7 : 1537-1545, 1998.

2. Farkas A, Kemény L, Szöny BJ, Bata-Csörgö Z, Pivarcsi A, Kiss M, Széll M, Koreck A and Dobozy A: Dithranol upregulates IL-10 receptors on the cultured human keratinocyte cell line HaCaT. Inflamm Res 50: 44-49, 2001.

3. Koo J and Arain S: Traditional Chinese medicine for the treatment of dermatologic disorders. Arch Dermatol 134: 1388-1393, 1998.

4. Tse TW: Use of common Chinese herbs in the treatment of psoriasis. Clin Exp Dermatol 28: 469-475, 2003.

5. Chen X, Yang L, Oppenheim JJ and Howard MZ: Cellular pharmacology studies of shikonin derivatives. Phytother Res 16: 199-209, 2002 .

6. Kourounakis AP, Assimopoulou AN, Papageorgiou VP, Gavalas A and Kourounakis PN: Alkannin and shikonin: Effect on free radical processes and on inflammation - a preliminary pharmacochemical investigation. Arch Pharm (Weinheim) 335: 262-266, 2002.

7. Hisa T, Kimura Y, Takada K, Suzuki F and Takigawa M: Shikonin, an ingredient of Lithospermum erythrorhizon, inhibits angiogenesis in vivo and in vitro. Anticancer Res 18: 783-790, 1998.

8. Xu Y, Xu X, Gao X, Chen H and Geng L: Shikonin suppresses IL-17-induced VEGF expression via blockage of JAK2/STAT3 pathway. Int Immunopharmacol 19: 327-333, 2014.

9. Yoon Y, Kim YO, Lim NY, Jeon WK and Sung HJ: Shikonin, an ingredient of Lithospermum erythrorhizon induced apoptosis in HL60 human premyelocytic leukemia cell line. Planta Med 65 $532-535,1999$.
10. Hsu PC, Huang YT, Tsai ML, Wang YJ, Lin JK and Pan MH: Induction of apoptosis by shikonin through coordinative modulation of the Bcl-2 family, p27 and p53, release of cytochrome $c$, and sequential activation of caspases in human colorectal carcinoma cells. J Agric Food Chem 52: 6330-6337, 2004.

11. Chang IC, Huang YJ, Chiang TI, Yeh CW and Hsu LS: Shikonin induces apoptosis through reactive oxygen species/extracellular signal-regulated kinase pathway in osteosarcoma cells. Biol Pharm Bull 33: 816-824, 2010.

12. Singh F, Gao D, Lebwohl MG and Wei H: Shikonin modulates cell proliferation by inhibiting epidermal growth factor receptor signaling in human epidermoid carcinoma cells. Cancer Lett 200: $115-121,2003$

13. Gong K and Li W: Shikonin, a Chinese plant-derived naphthoquinone, induces apoptosis in hepatocellular carcinoma cells through reactive oxygen species: A potential new treatment for hepatocellular carcinoma. Free Radic Biol Med 51: 2259-2271, 2011.

14. Chen CH, Lin ML, Ong PL and Yang JT: Novel multiple apoptotic mechanism of shikonin in human glioma cells. Ann Surg Oncol 19: 3097-3106, 2012.

15. Chen CH, Chern CL, Lin CC, Lu FJ, Shih MK, Hsieh PY and Liu TZ: Involvement of reactive oxygen species, but not mitochondrial permeability transition in the apoptotic induction of human SK-Hep-1 hepatoma cells by shikonin. Planta Med 69: $1119-1124,2003$.

16. Budihardjo I, Oliver H, Lutter M, Luo X and Wang X: Biochemical pathways of caspase activation during apoptosis. Annu Rev Cell Dev Biol 15 :269-290, 1999.

17. Elmore S: Apoptosis: A review of programmed cell death. Toxicol Pathol 35: 495-516, 2007.

18. Kinnally KW, Martinez-Caballero S and Dejean LM: Detection of the mitochondrial apoptosis-induced channel (MAC) and its regulation by Bcl-2 family proteins. Curr Protoc Toxicol Unit 2: 12, 2006.

19. Sun W, Zheng Y, Lu Z, Wang H, Feng Z, Wang J, Xiao S, Liu F and Liu J: LL-37 attenuates inflammatory impairment via mTOR signaling-dependent mitochondrial protection. Int J Biochem Cell Biol 54: 26-35, 2014.

20. Sun W, Zheng Y, Lu Z, Cui Y, Tian Q, Xiao S, Liu F and Liu J: Overexpression of S100A7 protects LPS-induced mitochondrial dysfunction and stimulates IL-6 and IL-8 in HaCaT cells. PLoS One 9: e92927, 2014.

21. Zhang Y, Qian RQ and Li PP: Shikonin, an ingredient of Lithospermum erythrorhizon, down-regulates the expression of steroid sulfatase genes in breast cancer cells. Cancer Lett 284: 47-54, 2009.

22. Dejean LM, Martinez-Caballero S, Manon S and Kinnally KW: Regulation of the mitochondrial apoptosis-induced channel, MAC, by BCL-2 family proteins. Biochim Biophys Acta 1762: 191-201, 2006.

23. Belsõ N, Széll M, Pivarcsi A, Kis K, Kormos B, Kenderessy AS, Dobozy A, Kemény L and Bata-Csörgõ Z: Differential expression of D-type cyclins in $\mathrm{HaCaT}$ keratinocytes and in psoriasis. J Invest Dermatol 128: 634-642, 2008.

24. Zhou LL, Lin ZX, Fung KP, Cheng CH, Che CT, Zhao M, Wu SH and Zuo Z: Celastrol-induced apoptosis in human $\mathrm{HaCaT}$ keratinocytes involves the inhibition of NF- $\mathrm{BB}$ activity. Eur J Pharmacol 670: 399-408, 2011.

25. Marija K and Larisa PM: The pole of apoptosis in psoriasis and lichen planus. In: Focus on Cell Apoptosis Research. Cho DW (ed). Nova Science Publishers, Inc., New York, pp233-240, 2007.

26. Okada $\mathrm{H}$ and Mak TW: Pathways of apoptotic and non-apoptotic death in tumour cells. Nat Rev Cancer 4: 592-603, 2004.

27. Gross A, Jockel J, Wei MC and Korsmeyer SJ: Enforced dimerization of BAX results in its translocation, mitochondrial dysfunction and apoptosis. EMBO J 17: 3878-3885, 1998.

28. Mao X, Yu CR, Li WH and Li WX: Induction of apoptosis by shikonin through a ROS/JNK-mediated process in Bcr/Abl-positive chronic myelogenous leukemia (CML) cells. Cell Res 18: 879-888, 2008.

29. Ahn J, Won M, Choi JH, Kim YS, Jung CR, Im DS, Kyun ML, Lee K, Song KB and Chung KS: Reactive oxygen species-mediated activation of the Akt/ASK1/p38 signaling cascade and p21(Cip1) downregulation are required for shikonin-induced apoptosis. Apoptosis 18: 870-881, 2013.

30. Min R, Tong J, Wenjun Y, Wenhu D, Xiaojian Z, Jiacai H, Jian Z, Wantao $C$ and Chenping $Z$ : Growth inhibition and induction of apoptosis in human oral squamous cell carcinoma Tca-8113 cell lines by Shikonin was partly through the inactivation of NF-kappaB pathway. Phytother Res 22: 407-415, 2008. 